\title{
Design of Harvesting Mechanism for Advanced Remote-Controlled Coconut Harvesting Robot (A.R.C.H-1)
}

\author{
Anoop Abraham*, M. Girish, H. R. Vitala and M. P. Praveen \\ Department of Mechanical Engineering, East Point College of Engineering and Technology, \\ Bangalore, India; a4abraham.88@gmail.com
}

\begin{abstract}
A.R.C.H (ark), robot is a coconut tree-climbing robot, designed specifically to harvest coconuts quickly, safely and efficiently. This robot is controlled remotely from the ground, and the operator can see what is happening on top of the tree on his remote-display. The A.R.C.H-1 robot prototype with its novel climbing and fastening mechanism is designed to climb a pole of varying diameter, which simulates a coconut tree. This manuscript deals with the design of a manipulator, with two unique modular end-effectors for the harvesting mechanism of A.R.C.H-1. The two robotic arms with three degrees of freedom are designed in such a way that it can be mounted on either side of the robot head. In addition, this paper also proposes two types of harvesting blades, suitable for the conditions on top of a coconut tree, which can be attached to the robotic arm.
\end{abstract}

Keywords: Agriculture Robot, Agrobot, Cocobot, Coconut-Tree Climber, Harvesting Robot

\section{Introduction}

Coconut picking is a tough, arduous work. The farmers in popular coconut cultivating countries are facing severe shortage of skilled workers for harvesting. In recent years, the number of coconut tree climbers has declined sharply, as those men who do this rigorous job of climbing 30 to 80 feet high above the ground, bare handed or with the aid of a rope, have broken away from their traditional role to take-up better paid jobs. Since the Green Revolution of the late 1960s, the harvesting of coconuts today has little changed from how it used to be done for generations. The job of coconut picking, which is perceived as risky and unglamorous, does not attract youngsters either, even though the remuneration is fairly good in local standards.

Though there exist few tree climbing machines today, by any measure, climbing for coconuts is hard work. Not only does a climber have to scale the tree and reach out among the fronds and cut the coconut, but they also have to instinctively learn which nuts are ripe enough to be harvested. Besides, those few climbers who still remain in the business of coconut picking, claims higher wages.

There are several industries, which depend on raw coconuts, for its processing and manufacturing of various value-added products. The inflorescence sap of coconut palms used to produce Neera (sweet toddy or palm nectar), desiccated coconut and its powder, packed coconut milk, coconut cream, coconut milk powder, tender coconut water, vinegar, nata-de-coco, etc. are few of them. The growing market for all coconut products is waking up even in the American and European countries that have never used coconut in their cuisine. Hence, any impact in coconut cultivation

${ }^{*}$ Author for correspondence 
sector will adversely affect these industries and their markets.

In such situation there is genuine need to develop an efficient coconut-harvesting robot to revive the coconut industry. A.R.C.H robot (Advanced Remote-controlled Coconut Harvester), as illustrated in Figure1, was designed and developed to tackle the issues faced by coconut farmers around the world today. The robot is light in weight and simple, controlled from the ground, fast, easy to operate and is portable to carry it along in large plantations, at the time of harvesting.

\section{Motivation}

In traditional harvesting method on reaching the top of a coconut tree, the climber taps the nut in the lowermost bunch with its harvesting knife to test its maturity. If he is satisfied, he cuts the bunch at the base of the stalk when it drops down to the ground. The climber also cleans the crown and removes the dry leaves, sheaths and spathes. In some places where the trees are not tall, cutting the coconut bunches with a knife, attached to a long bamboo pole, does the harvesting. It seems quite simple to climb the coconut palm in order to stay beneath the leaf crown to harvest fruits and cut old leaves. But it proves much more challenging to climb up the coconut leaf crown in order to reach the young inflorescences for making controlled pollinations with bagging for research purposes, and for harvesting the toddy (the sweet sap from coconut inflorescences), which serves to produce sugar, vinegar, wine and spirits. This paved the way to the proposed robotic harvesting method, where it can peep and reach out to any remote nook and corner at the top of the tree, of the operator's choice.

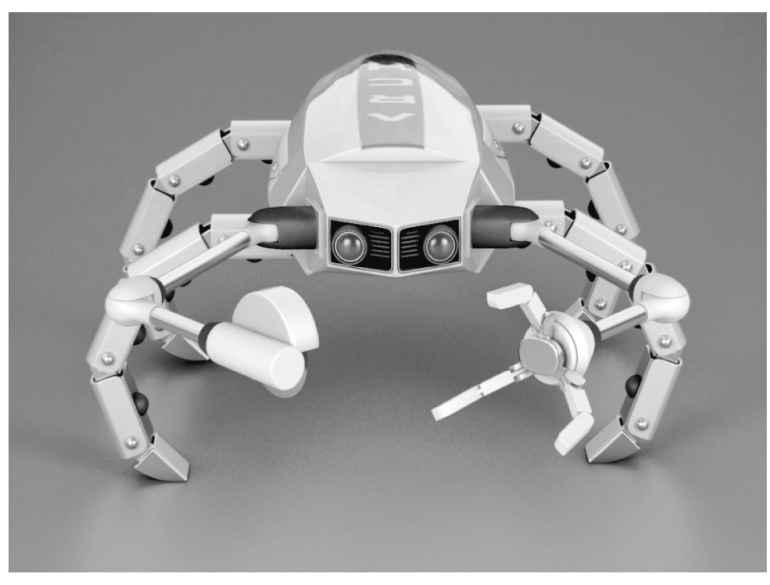

Figure 1. 3D rendered model of A.R.C.H robot.

\section{Background Research}

There are several countries working effectively on the development of harvesting robots. Most research work has been done to perform plant inspection, transportation, grafting and especially harvesting of fruits and vegetables in horticulture. A few coconut tree-climbing robots have been developed, especially in South Asia for harvesting coconuts. But those projects primarily focus on the climbing mechanism of the robot. Prakasan Thattari from the South Indian state of Kerala designed and developed a prototype of Coconut Tree Climbing Robot. It is remotely controlled from the ground and works on a rechargeable battery. The robot has a long retractable arm with a sharp cutting blade as end-effector at one end and a twisting device at the other end. Its design is not compact or agile, and is less maneuverable. A team of graduate students from Amrita Vishwa Vidyapeetham University, Kerala has designed a coconut tree climber called CocoBot ${ }^{1}$. Its manipulator has three mutually orthogonal rotational joints, which are controlled using three separate motors. The elbow is modeled with single rotational joint and wrist as end effecter.

Using robotic arms with modular end-effectors in harvesting robots is necessary, since it is quite challenging for a robot to reach around a tree or plant to pick fruits and vegetables. The igus Robolink shown in Figure 2, with multiple-axis joint system, was developed by igus ${ }^{\circledR}$ Germany and released in 2011. It is composed of a set of lightweight plastic joints, metal tubes, drive units, cables and end attachments that are meant to work together to produce an infinite variety of low cost and low maintenance robot arms.

The Robolink system has a large number of example applications ranging from swimming robots, to pick and place industrial robots, to humanoid robots.

A few good examples of the Robolink system in use can be found in humanoid robots, all of which extend the maximum number of DOF afforded by the Robolink via complex grippers and hands.

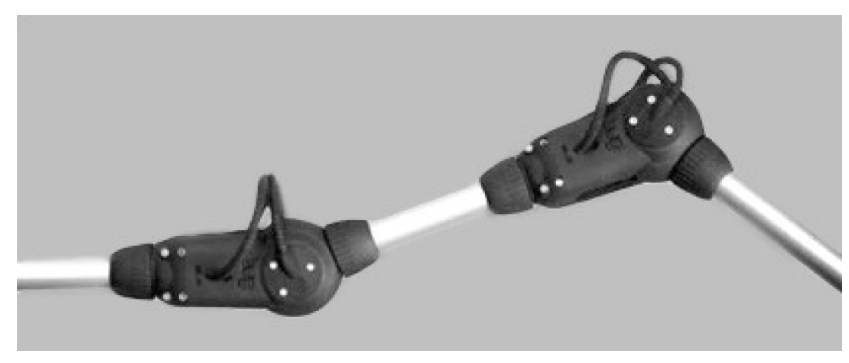

Figure 2. Igus Robolink. 
A substantial number of human labors are usually needed for fruit harvesting. The workers often must pick fruit by hand, being gentle to avoid bruising it. But a growing number of companies and researchers are developing robotics to address those challenges. Robotic Harvesting LLC, based in the Los Angeles, is testing a product for strawberry fields. Its prototype as shown in Figure 3, rolls through the fields with a camera that identifies berries and a robotic arm that picks them. The arm's foampadded claw, which can pick a strawberry every five seconds, places them on a conveyor to be carried to the top of the vehicle and placed in boxes.

Energid Technologies Corporation, of Cambridge, Massachusetts, is working on a truck-mounted prototype with a large hydraulic arm for harvesting - orange. Machines already exist that grab orange trees and shake their fruit loose. But Energid's approach is designed to do less damage to the trees and doesn't require low branches to be removed before use. The University of Sydney is developing a robot illustrated in Figure 4, which can recognize fruit. Vision-based fruit detection technology can

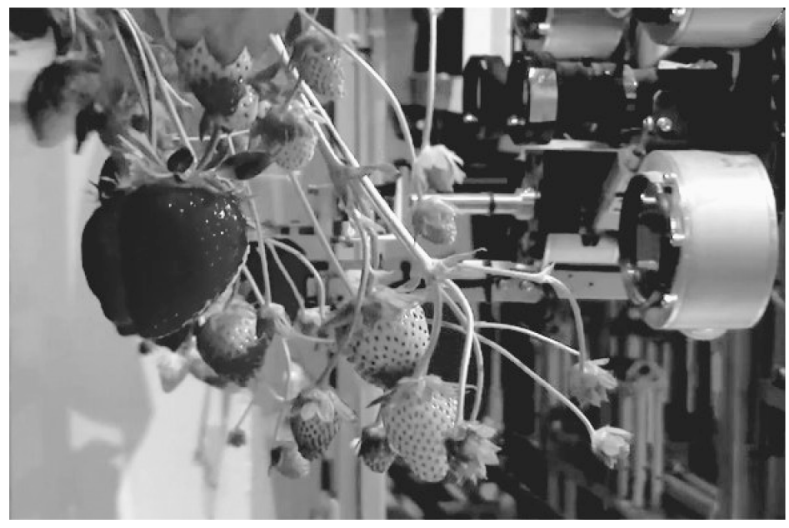

Figure 3. Strawberry-picking robot.

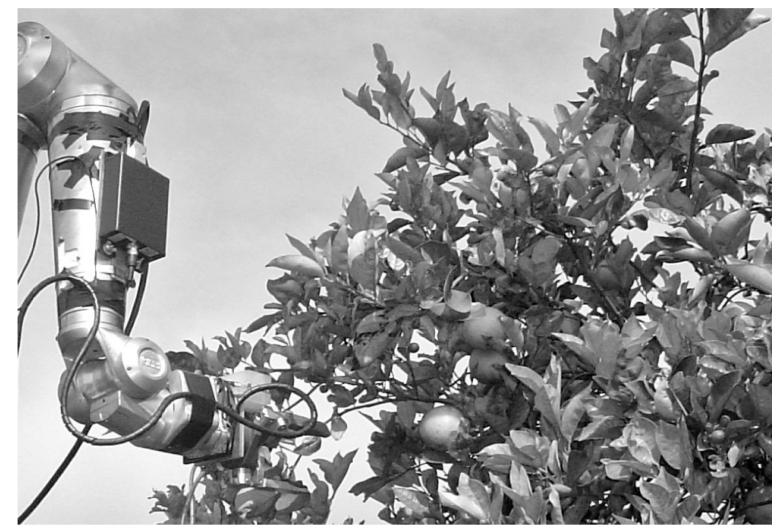

Figure 4. Vision based fruit-picking robot. easily be tailored for other applications such as on-tree yield monitoring, crop health status monitoring, disease detection, maturity detection and other operations, which require vision as a sensor.

\section{Design of Harvesting Mechanism for A.R.C.H-1}

Arms are types of jointed robot manipulator that allow robots to interact with their environment ${ }^{2,3}$. Basically, a robot arm consists of joints, links, and end-effectors. The joints are where the motion in the arm occurs. Many have onboard controllers or translators to simplify communication, though they may be controlled directly or in any number of ways. The robot arms can be autonomous or controlled manually and can be used to perform a variety of tasks with great accuracy. The robotic arm can be fixed or mobile and can be designed for industrial, agricultural or home applications. The robotic arms can be characterized by their mechanical structure. Cartesian (also known as Gantry) robots have three joints that are coincident with the standard X-Y-Z Cartesian axes. Cylindrical arms have any number of joints that operate on a cylindrical axis, normally rotating about one fixed rod. Spherical (polar) arms are those with joints that allow it full rotation throughout a spherical range. SCARA robots have two parallel rotary joints to allow full movement throughout a plane, typically for pick-and-place work. Articulated robots are usually used for complex assembly operations, and consist of three or more rotary joints. Parallel robots have three concurrent prismatic or rotary joints, and allow for tilting of heavy or sensitive platforms ${ }^{4}$.

The harvesting mechanism of A.R.C.H-1 robot consists of two arms as illustrated in Figure 5, each with different end-effectors, mounted on either side of the robot head. These arms also help to balance the weight of the robot preventing it from toppling sideways. Both harvesting arms have 3 Degrees of Freedom.

The base of the arm has 2 DOF (Degrees of Freedom), both oscillation and swivel, and 1 DOF (Degrees of Freedom) at the joint, which enable it to oscillate. One end-effector is a sharp high speed rotating steel cutting blade for harvesting the desired coconuts. The other endeffector has 3 fingers, on a rotating spindle, which helps the coconut bunch to be pushed away during harvesting. This avoids the possibility of coconuts falling on to the robot itself and it also helps to push away or move any obstacle in its path. The aluminium tubes of each 
end-effector arm are $150 \mathrm{~mm}$ (Tube x1) and $100 \mathrm{~mm}$ (Tube $\mathrm{x} 2$ ) in length respectively.

In A.R.C.H-1 robot, the arm is fixed to the robot's head by shoulder joint. The joint is flexible and joins two separated links. There were few challenges involved in designing a robotic harvesting mechanism, especially dedicated for coconut harvesting. Both modular robotic arms should balance the weight of the robot. Designing a harvesting arm with two different end-effectors, is another challenge. During operation, the arm is subjected to vibrations when the high speed cutting blade rotates. These vibrations can cause the robot to tople sideways and even lose the grip if not properly fastened. Design of a fully functional end-effector with human like fingers on a rotating spindle is a major hurdle.

\subsection{Design Constraints}

\subsubsection{Mounting the Arms}

Several constraints were encountered in achieving the design goals. Mounting the arms on the robot head posed the issue of finding sufficient space. The arm also needs to be interfaced with the robot's power supply and camera modules. As explained in the proof of concept experiment above, the workspace of the arm with respect to both the control unit and robot head needed to be accommodated.

\subsubsection{Passive End-effectors}

Another important design constraint involved is the swapping of end-effector. The end-effector needs to be

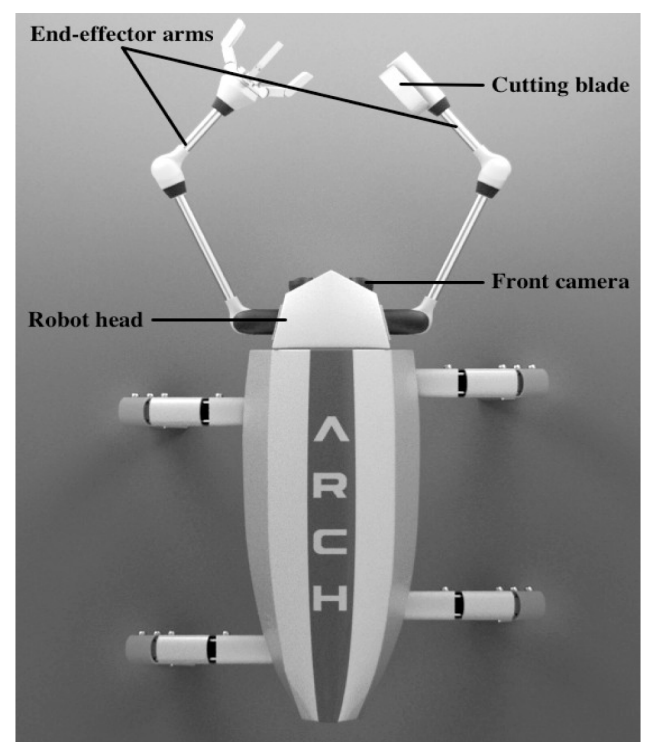

Figure 5. Harvesting mechanism of A.R.C.H-1. modular, easy to remove and replace the existing gripper. This end effector also needs to be versatile enough to work for multiple tasks including tree maintenance.

\subsection{Design Solutions}

Harvesting arms need to be mounted such that the endeffectors on both the arms could reach the target position. Possible mounting solutions involved welding another aluminium metal sheet to the front end of the base. This allows sufficient space to mount the arms, motors and camera module. Commercial Grade aluminium sheet of $2 \mathrm{~mm}$ thickness of length $10.5 \mathrm{~cm}$ was used as the extension sheet.

This initial design involved using a single camera but during the development process, a second camera was installed to the head, allowing a wider camera-viewing angle. The second version of the design also considered the possibility of small objects on the tree, such as the sheaths and spathes, which may fall over the camera module. Hence both the cameras were positioned in the front in such a way that, they are mounted on a specific angle. Now the objects falling over the camera modules may slide away and fall down without blocking the view.

\section{End-effectors of A.R.C.H-1}

The first and foremost task of a harvesting robot is to design the suitable end-effector. Each particular agricultural product has a pecular way of harvesting method, thus requires specialized end-effector ${ }^{5}$. The harvesting mechanism of A.R.C.H-1 robot consists of two distinct end-effectors as illustrated in Figure 6. One end-effector has 3 human like fingers, on a rotating spindle, which helps the coconut bunch to be pushed away and clear any obstacle like dry leaf in the robot's path, during climbing

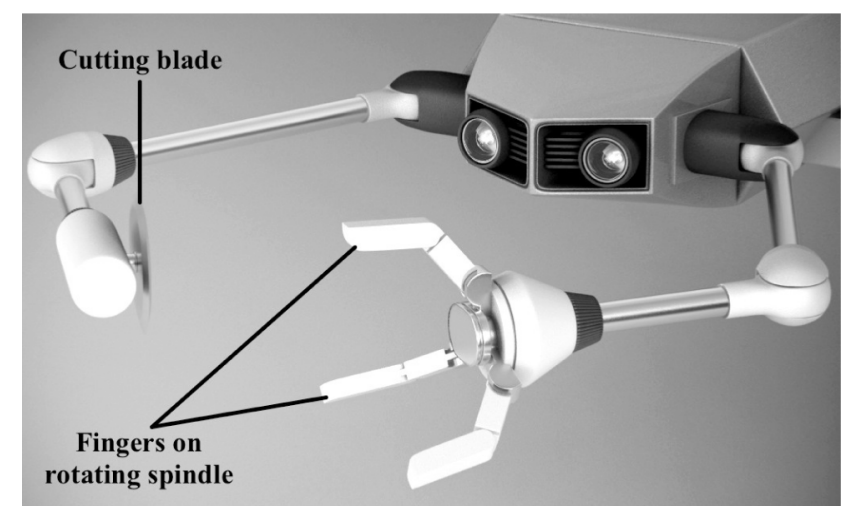

Figure 6. End-effectors of A.R.C.H-1. 
and harvesting. This end effector has two components: the coconut holding fingers and the rotating spindle.

The finger bending mechanism of the end-effector includes a spring plate, wire, and solenoid. One spring plate is inserted into each of the three fingers, with a wire connecting the tip to the solenoid. When the solenoid is magnetized, it pulls the steel wire on the inside of the finger, drawing the tip in and forcing the spring plate to bend. This causes the two sections of the fingers to curl similarly to human fingers when gripping something. Limit switches that control the finger curving extent were inserted into the base of the fingers. When the three fingers curl together, the opening of the tip of the endeffector shrinks, enhancing its grip ${ }^{6}$.

The main advantage of this end effector is its contact with the coconuts. It is feasible for the end-effector to hold a portion of an entire bunch of coconuts only because of the high tensile strength between the stalk and the coconuts. The term coconut can refer to the entire coconut palm, the seed, or the fruit, which, botanically, is a drupe, not a nut. Two layers cover a coconut, the outer layer is the thick coconut husk and the inner layer is the thin hard coconut shell. Hence it is highly unlikely that a coconut will get damaged in a free fall from a height of 30 to 80 feet. Once the cutter blade cuts the stalk, the finger like end-effector pushes the coconut bunch further away, to avoid any contact between the free falling coconuts and the robot. This will avoid the possibility of damaging the robot while harvesting.

The other end-effector of the harvesting mechanism of A.R.C.H-1 robot is a sharp circular high-speed rotating blade, for harvesting the desired coconuts. This rotary blade is intended to cut the stalk of the ripe coconut bunch. The possibility of using a modular chainsaw for harvesting is also discussed in this paper.

\subsection{Circular Blade Model}

A rotary stainless steel cutter blade, cuts the stalk of a ripe coconut bunch on the tree. The video feedback from the cameras in the front will allow the operator on the ground to select and position the blade along the stalk. The blade has a safety cover which partially exposes the blade. It protects the chips from shattering on to the camera modules. Figure 7 shows the circular cutting blade endeffector of $10 \mathrm{~cm}$ diameter, which consists of a DC motor to drive the blade. The motor blade assembly is mounted on a manipulator of the harvesting mechanism.

\subsection{Chainsaw Model}

Harvesting is an intricate task in which the end-effector will come in contact with the coconuts. It should be pointed that only the ripe coconuts should be harvested and other coconuts on the tree should be free of damage. This is one of the major challenges of end-effector development.

In a coconut tree with a dense crown, it will hard for the rotary blade end-effector to penetrate through the coconut bunches and cut exactly the Stalk of the ripe bunch as illustrated in Figure 8. Though the safety cover on the blade avoid the contact between the blade and objects around it, circular blade may not be practical in a coconut tree with a dense crown. Hence a chainsaw endeffector as shown in Figure 9 is proposed, which should overcome the challenges mentioned above. Chainsaw is a mechanical saw, which is powered by electricity. Each segment in this chain, of the chainsaw, features small sharp cutting teeth. Each tooth takes the form of a folded tab

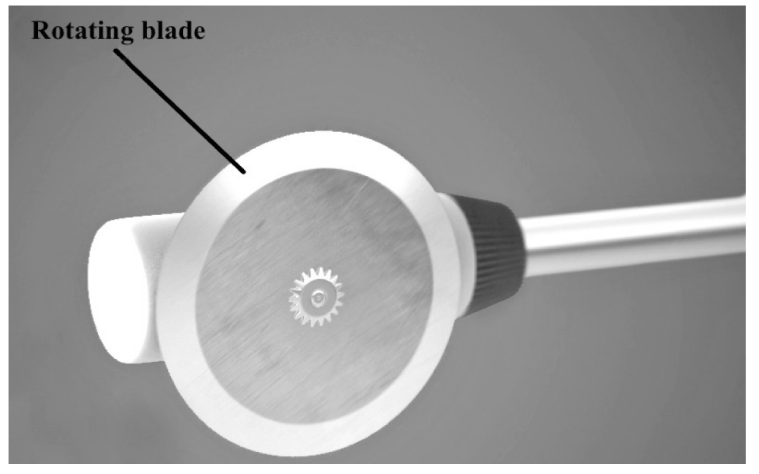

Figure 7. High speed rotating blade.

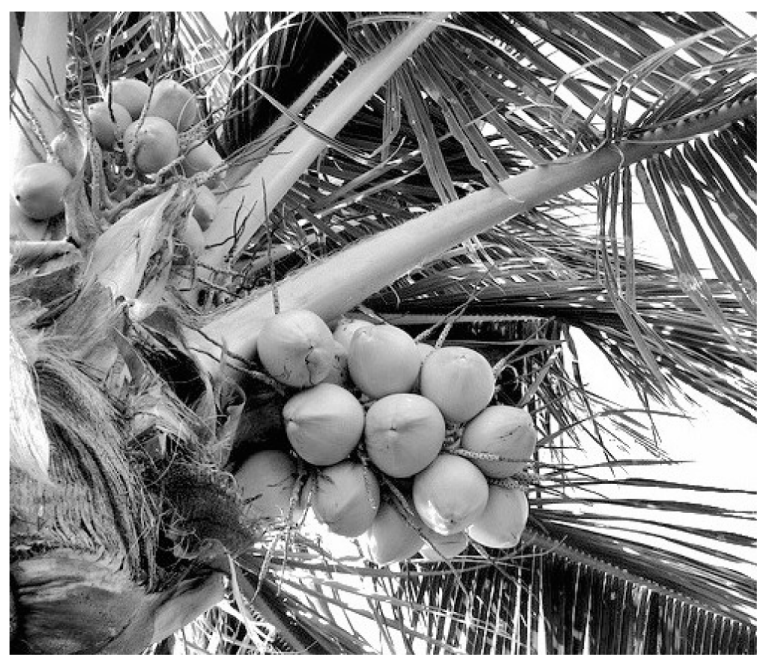

Figure 8. Ripe coconut bunch on a coconut tree. 


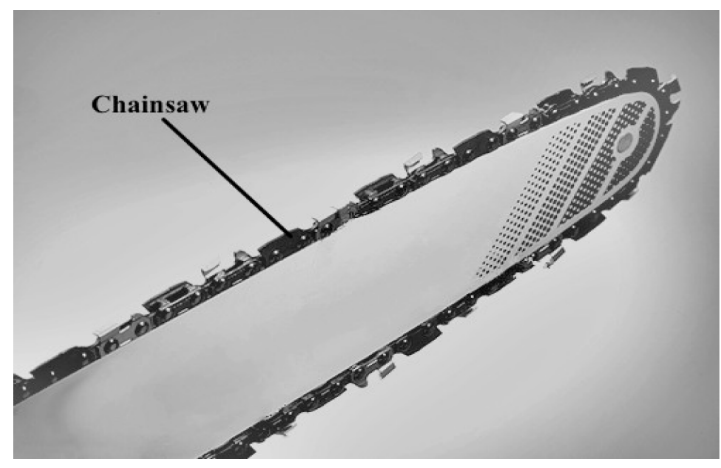

Figure 9. Chainsaw end-effector.

of chromium-plated steel with a sharp angular or curved corner and two cutting edges, one on the top plate and one on the side plate. Left-handed and right-handed teeth are alternated in the chain.

Kkaarrlls, a platform for a collection of objects resulting from coursework, realized by European designers at Karlsruhe University of Arts and Design, in partnership with Berlin-based company Echtwald, a stackable 7Xstool was cut straight from a tree trunk. The robot is a standard Kuka industrial robot with a chainsaw end effector. By programming, the high precision robot can curve a tree trunk with millimeter accuracy into 2-3 usable stools. Similar end-effector system can be adapted for harvesting coconut, which ensures precision and accuracy.

\section{Conclusions and Future Scope}

The trials were conducted using manually controlled A.R.C.H-1 robot prototype, which was made to climb a rough surfaced pole, of varying diameter, instead of a tree. Although, the number of trials was not sufficient to warrant the reliability of this prototype, it was enough to show that it could harvest the coconuts. In future we intend to increase the degrees of freedom of the robotic arms by implanting more stepper or servo motors and equip the robotic arm with sensors to detect whether the coconuts are ripe or not. By making the robot autonomous in detecting ripe coconuts, the controller on the ground, will have to only focus in maneuvering the robot on the coconut tree.

\section{Acknowledgement}

We do appreciate and thank Dr. S. K. Harisha, Associate Professor of R. V. College of Engineering, for the key discussions on the practicality and implication of the endeffector design. His commitment to excellent delivery made a huge impact. And here goes our sincere gratitude to Jobin Philip, A. S. Vysakh and Roy Skaria, who are the members of the A.R.C.H team, Mac Pro Technologies, Sri Sai Engineering, and then our friends and families, for their bits and pieces of support and contributions.

\section{References}

1. Megalingam RK, Tom CK, Harikrishna MT, Raidu VM. CocoBot: A kinect based coconut tree climber. Int J Appl Eng Res. 2012; 7(11): 1497-502.

2. Md Anisur R, Alimul HK, Tofayel A, Md Mohsin S. Design, analysis and implementation of a robotic arm - The animator. AJER. 2013; 2(10):298-307.

3. Emanuele C, Stefano R, Nicola V, Irene S, Panagiotis KA, Pierpaolo V, Fabrizio V, Maria CC, Kostas JK, Paolo D. Design and development of a novel robotic platform for neuro-robotics applications: the NEURobotics ARM (NEURARM). Adv Robot. 2008; 22:3-37.

4. Rama K, Sowmya B, Sastry ASCS, Bhanu PS, Gokul SA. Design and implementation of a robotic arm based on haptic technology. IJERA. 2012; 2:3098-103.

5. Wan IWI. Research and development of oil palm harvester robot at universiti putra malaysia. Int J Eng Tech. 2010; 7(2):87-94.

6. Yi-Chich, Pen-Yuan, Suming. Development of the endeffector of a picking robot for greenhouse-grown tomatoes. The 43rd International Symposium on Robotics (ISR2012); 2012 Aug 29-31; Taipei, Taiwan. 